\section{New education academy opens}

Dentsply Sirona has officially opened a new venue which joins many others worldwide to form part of the Dentsply Sirona Academy. The opening of the new facility took place on 7 June at Dentsply Sirona's UK Headquarters in Weybridge.

The Dentsply Sirona Academy exists to address the continuing educational needs of all dental professionals, providing worldwide access to evidence-based, scientifically sound, theoretical and practical content. The Dentsply Sirona Academy provides over 11,000 courses annually in more than 80 countries, delivering education to approximately 350,000 dental professionals every year, covering an extensive range of clinical, technical and practice excellence programmes.

The Dentsply Sirona Academy in Weybridge is the embodiment of Dentsply Sirona's educational philosophy. This multi-use facility will showcase products and equipment in a clinical setting and allow customer training to take place. In addition, it will provide a UK base for Dentsply Sirona’s extensive in-house clinical and technical education programmes that enable dentists and technicians to not only hear

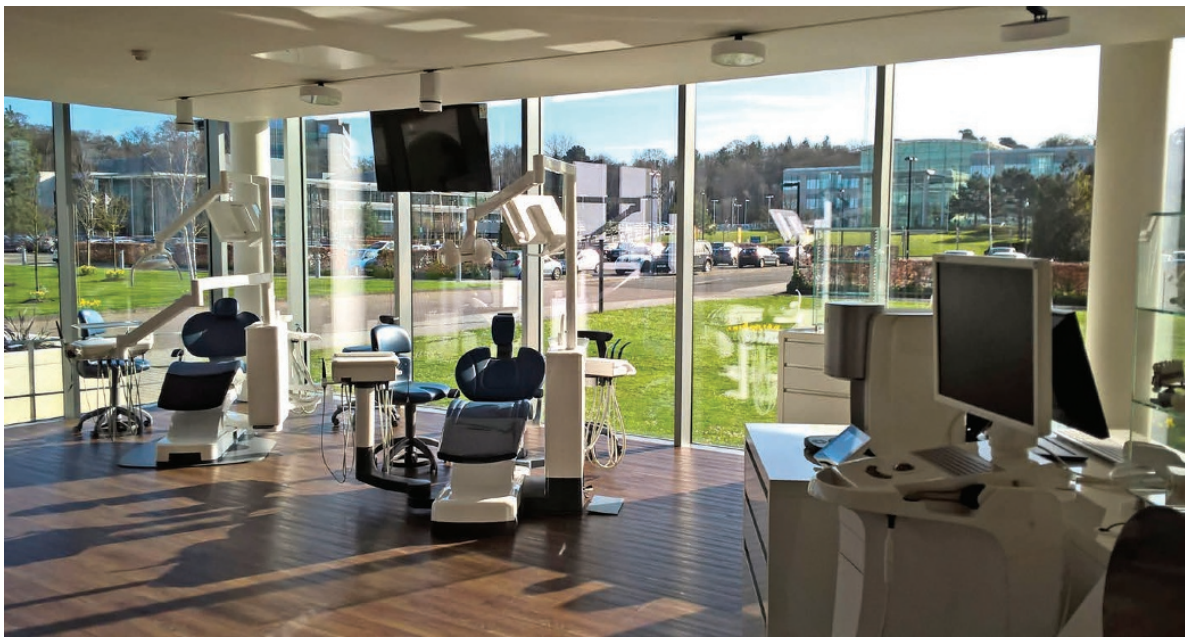

about how an integrated solution can improve their workflow, but to experience it first-hand.

The specially designed facility incorporates a Clinical Skills Suite featuring an eight station simulation clinic complete with phantom heads. A showroom includes treatment centres, handpieces and digital radiography, including fully functioning examples of the Galileos cone beam imaging system and Orthophos XG3D. Having this equipment on site enables visitors to appreciate the streamlined efficiency that can be gained by adopting an integrated workflow.

\section{New emergency eyewash station}

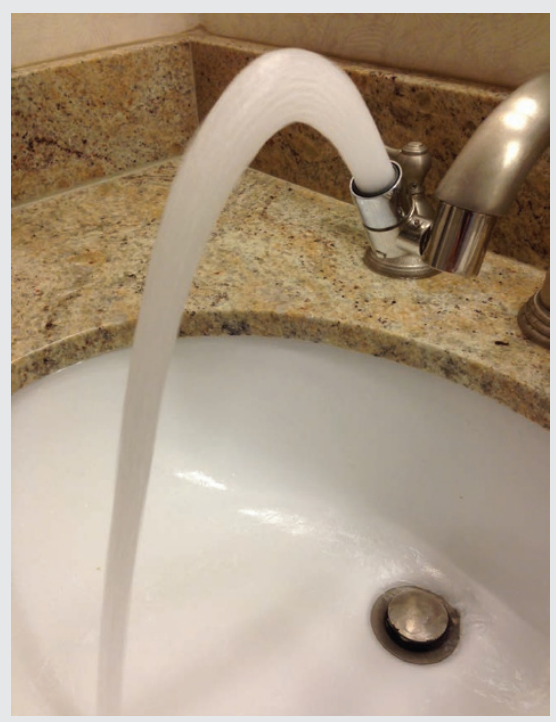

TAUB Products, a long-time manufacturer of dental laboratory and restorative products, has introduced a low-profile emergency eyewash station that adapts to a wide range of office and laboratory faucets. The new station provides instant access to a single aerated water jet, flushing the eyes and washing the face of chemical or particulate contamination.

The unique design allows water to flow normally downward for washing hands, and during an emergency, the user can quickly rotate it upwards, providing a soft stream of water that will wash the face. The user places the water stream on the brow of the nose and both eyes receive an effective rinse.

Setting this apart from dual-jet designs, the key features of the product include a single rotating nozzle to wash eyes and face, a low-profile modern design that fits most standard faucets, a flow rate of 1.2 gallons per minute, which exceeds ANSI standards, and a design that prevents standing water, thus reducing the chance for mould development.

TAUB Products is celebrating its $65^{\text {th }}$ year in business. TAUB provides innovative, high-quality solutions for dental professionals. For more information on TAUB and its products go to www. taubdental.com.
These benefits are exhibited across specific disciplines including, restorative dentistry, implants, endodontics and orthodontics.

A 30-seat classroom style lecture room can be rapidly transformed to accommodate $60+$ in theatre style, providing a flexible resource that can accommodate almost any training or education requirement. And of course, stateof-the-art audio visual equipment makes these facilities amongst the finest in the UK.

See more at: www.dentsply.com/en-uk/ education/dentsply-sirona-academy.html.

\section{One less thing to worry about}

Posterior restorations can be challenging; there are lots of things that the practitioner has to worry about if they want treatment to be a success.

Fortunately, Filtek One bulk fill restorative from $3 \mathrm{M}$ Oral Care is not one of those things. Here's why:

- Increased opacity for improved aesthetics

- One-step placement at up to $5 \mathrm{~mm}$ without sacrificing the aesthetic result

- Unique nanotechnology that allows for durability and polishability

- Excellent handling and sculptability

- Five VITA shades for better aesthetic matches.

Filtek One bulk fill restorative is one less thing for you to worry about. To find out more, contact the friendly $3 \mathrm{M}$ Oral Care team today.

For more information, call 0845602 5094 or visit www.3Mespe.co.uk.

$3 \mathrm{M}$ and Filtek are trademarks of the 3M Company. 